ILL-(TH)-94-4

Feb. 1994

\title{
LOOKING FOR THE LOGARITHMS IN FOUR-DIMENSIONAL NAMBU-JONA-LASINIO MODELS
}

\author{
Seyong KIM \\ High Energy Physics Division, Argonne National Laboratory \\ 9700 S. Cass Avenue, Argonne, Il 60439 \\ Aleksandar KOCIĆ and John KOGUT \\ Loomis Laboratory of Physics, University of Illinois \\ 1110 W. Green St., Urbana, Il 61801-3080
}

\begin{abstract}
We study the problem of triviality in the four dimensional Nambu-Jona-Lasinio model with discrete chiral symmetry using both large- $N$ expansions and lattice simulations. We find that logarithmic corrections to scaling appear in the equation of state as predicted by the large- $N$ expansion. The data from $16^{4}$ lattice simulations is sufficiently accurate to distinguish logarithmically trivial scaling from power law scaling. Simulations on different lattice sizes reveal an interesting interplay of finite size effects and triviality. We argue that such effects are qualitatively different for theories based on fundamental scalar rather than fermion fields. Several lessons learned here can be applied to simulations and analyses of more challenging field theories.
\end{abstract}




\section{Introduction}

The Nambu-Jona-Lasinio (NJL) model [1] describes the dynamics of massless fermions that have a contact interaction. The theory has two phases. At weak couplings fermions are massless, while at strong couplings they acquire dynamical mass due to spontaneous chiral symmetry breaking. The transition from a massless to a massive phase is accompanied by the appearance of light scalar (and pseudoscalar) bound states. Through the exchange of these composites an effective interaction develops over physical scales and the cutoff disappears from the model [1]. The original theory, although not renormalizable in perturbation theory, becomes renormalizable and appears to have the same low energy limit as a linear $\sigma$-model [2]. Since the effective interaction is of the Yukawa type, it is important that, measured in physical units, the mesons retain a finite extent in order to ensure an interacting low energy limit. This is possible to achieve below four dimension $[3,4]$, but for $d>4$, the mesons are always pointlike in the continuum limit and the corresponding theory is free. In the marginal dimension, $d=4$, there are calculations and analyses supporting triviality as well $[3,5]$, but a rigorous demonstration is lacking.

The reason for triviality in the NJL model is different from that in the more familiar and more extensively studied case of $\phi^{4}$ theory [6]. The essence of the chiral phase transition is to tune the coupling in such a way to allow for the formation of bound states. Once bound states appear in the spectrum, everything else related to chiral symmetry breaking follows naturally [7]. Keeping the size of the bound state finite, on the other hand, is a matter of balancing the attraction due to the interaction and the zero-point repulsion due to the kinetic energy. Above four dimension, this is not possible to achieve since the short-distance attraction becomes progressively stronger with increasing dimensionality and collapsing bound states always lower the energy.

In four dimension, however, the attraction and the repulsion scale identically and the physics is driven by whatever is left over after their cancellation. So, the problem of triviality in four dimension amounts to more than simple power counting and a decisive answer requires a careful study. The technical difficulties lie in the fact that power counting in the four dimensional case is modified by logarithmic corrections and these logarithms are notoriously difficult to establish both theoretically and numerically. There are several techniques, such as large- $N$, $\epsilon$-expansions or perturbation theory, that can provide some hints of how the logarithms enter the game $[3,8,9]$. In the NJL model the physics of triviality is intrinsically nonperturbative due to the presence of bound states and chiral 
symmetry breaking - the weak coupling phase is symmetric and the Goldstone physics is not accessible to perturbation theory. Thus, the only analytic method that can be of some utility in the context of NJL models is the $1 / N$ expansion which we will use as a guide in analyzing the data.

In the past the NJL model has captured considerable attention and has stimulated activities in a wide range of contexts, from purely theoretical [3,10-12] to practical model building, such as nuclear physics and the intermediate energy sector of $Q C D[13,14]$, technicolor [15] and the top condensate model [16], to mention a few. Most of the studies have been performed in the large- $N$ limit, for the reasons mentioned above, and systematic studies of the $1 / N$ corrections have been carried out. The $1 / N$ expansion indicates that below four dimension the theory is renormalizable [3,8], whereas for $d \geq 4$ it is trivial. There is little doubt that this changes at low- $N$, but no proof outside of the $1 / N$ expansion exists. In that sense we have undertaken this project in order to gain some insight and develop some criteria to find logarithms in a theory that is known to have them.

Recently several groups have carried out numerical simulations of various versions of the NJL model, at [16] and below four dimension [18,19], but the problem of triviality in four dimension has not been tackled intensively before. In this paper we want to address precisely this issue with an emphasis on how the logarithms should be tracked down. We should point out that we do not expect any surprises as far as the large- $N$ predictions are concerned and, in that sense, we anticipate the outcome that the theory is trivial. Our main motive is to see, knowing the answer, with what degree of confidence we can establish it numerically. Our main efforts will be concentrated on the equation of state (EOS) and the logarithms that accompany critical scaling. We have been encouraged to undertake this project by the recent observation that the logarithms in models with composite mesons, (therefore the NJL model), show up in a qualitatively different way than in theories with elementary scalars, like $\phi^{4}[20]$. Interestingly, the logarithmic behavior from our simulations on large lattices $\left(16^{4}\right)$ agrees with the composite meson case, as expected. However the logarithmic behavior from the simulations on the smaller lattices $\left(8^{4}\right)$ is consistent with the elementary scalar case. This apparent disagreement between two lattice simulation results must be due to the systematics of finite size effects and understanding the reason behind it will increase our confidence in deciding whether a particular lattice result is tainted by finite size effects. Our efforts include some technical aspects of this problem as well. In particular, we will show how to distinguish power 
law scaling from mean field scaling modified by logarithmic corrections. This problem is "reversed" from searches for a nontrivial fixed point in other models. As will be discussed in section 4, when we discuss the lattice data, the logarithms in the NJL model reveal themselves in a clear and unambiguous way. Especially interesting, in this context, is the manner in which the power law hypothesis fails.

There are important lessons to be learned from this exercise alone. They concern numerical simulations of theories with nontrivial fixed points for which no analytic tools are available. On a technical level, establishing the logarithms (therefore triviality), is a more demanding job than diagnosing their absence in theories that are not trivial.

Setting our goal as "logarithm hunting", we have chosen to simplify our task as much as possible. For that reason, we decided to work with $N=12$ flavors in order to remain close to the large- $N$ limit. This turned out to be a profitable decision for another reason. Tunneling is suppressed at large- $N$, so we could work relatively near the model's critical point without suffering from this particular finite size effect. Also, the choice of discrete chiral symmetry, instead of continuous chiral symmetry enables us to work in the chiral limit and avoid the extrapolations to zero bare mass which complicate other studies in the field.

The layout of this paper is as follows. In Sec.2 and 3 we solve the model in leading large- $N$ order and identify the logarithms of triviality in the equation of state, susceptibility, and specific heat. These results are contrasted to the analogous quantities in $\phi^{4}$. In Sec. 4 we present the simulation data and fits on $16^{4}$ and $8^{4}$ lattices. We shall see that the $16^{4}$ lattice simulations expose the logarithms of the large- $N$ expansion with considerable precision. The $8^{4}$ data is strongly distorted by finite size effects which in some cases obscures the logarithms in curious ways. In Sec.5 we discuss our results and the lessons we have learned which can be applied to studies of more challenging field theories.

\section{Large- $N$ limit}

In this and latter sections we review the large- $N$ limit of the NJL model. The emphasis will be on the scaling violations and how they enter the EOS and expressions for other thermodynamic quantities. In order to facilitate the large- $N$ expansion, the four-fermi lagrangian can be written in the form of a Yukawa theory

$$
L=\bar{\psi}(-i \not \partial+m+g \sigma) \psi-\frac{1}{2} \sigma^{2}
$$


The original, four-fermi, lagrangian is recovered after integrating over the $\sigma$ field. Since $L$ is quadratic in fermionic variables, we can integrate them out and obtain the effective action

$$
S_{\text {eff }}=\frac{1}{2} \int_{x} \sigma^{2}-N \operatorname{tr} \ln (-i \not \partial+m+g \sigma)
$$

In the large- $N$ limit the dominant configurations are obtained by the saddle point method. The resulting equation of state (EOS) is the minimum condition $\sigma-N g \operatorname{tr} \int_{q} 1 /(\not q+m+$ $g \sigma)=0$. After identifying the fermion mass as $\Sigma=m+g \sigma$, the minimum condition reads

$$
\Sigma=m-g^{2}<\bar{\psi} \psi>
$$

The curvature of the effective action is the inverse susceptibility

$$
\frac{\delta^{2} S_{e f f}}{\delta \sigma^{2}}=\chi^{-1}=1-4 g^{2} \int_{q} \frac{1}{q^{2}+\Sigma^{2}}+8 g^{2} \int_{q} \frac{\Sigma^{2}}{\left(q^{2}+\Sigma^{2}\right)^{2}}
$$

The appearance of soft modes at the critical point is manifested in the divergence of the susceptibility and the vanishing of the fermion mass. Setting $\Sigma=0$ in eq.(2.4) gives $1-4 g_{c}^{2} \int_{q} 1 / q^{2}=0$. With this expression for $g_{c}$, the EOS can be recast in the form

$$
\frac{m}{\Sigma}+t=4 g^{2} \int_{q} \frac{\Sigma^{2}}{q^{2}\left(q^{2}+\Sigma^{2}\right)}
$$

where $t=g^{2} / g_{c}^{2}-1$. This is a convenient form for extracting the critical indices since one need only count the infrared divergences of the right hand side. The indices associated with the EOS are defined as [21]: $\left\langle\bar{\psi} \psi>\sim t^{\beta}\right.$ and $\chi \sim t^{-\gamma}$ at $m=0$, and $\left\langle\bar{\psi} \psi>\sim m^{1 / \delta}\right.$ at $t=0$. Since there are only two fields the system responds to, only two out of three indices are independent. They are related by the scaling relation $\beta(\delta-1)=\gamma$. Above four dimension, the integral in (2.5) is infrared finite, and the exponents do not change with dimensionality - they have the mean field values: $\beta=1 / 2, \delta=3, \gamma=1$. Below four dimension the right hand side scales as $\Sigma^{d-2}$ resulting in nongaussian critical behavior with indices $\beta=1 /(d-2), \delta=d-1, \gamma=1$. In four dimension, the integral is logarithmically divergent giving rise to scaling violations. The EOS in this case reads

$$
\frac{m}{\Sigma}+t=a g^{2} \Sigma^{2} \ln \frac{b}{\Sigma^{2}}
$$

where $a$ and $b$ are constants, and the cutoff is set equal to one. Using a momentum cutoff gives $a=1 / 4 \pi^{2}$ and $b=1$. In the chiral limit, the fermion mass scales as the order 
parameter, $\Sigma \sim<\bar{\psi} \psi>$. At the critical point $\Sigma=\left(m+C m^{1 / \delta}\right)$, but since $\delta>1$, the linear term is subleading and the scaling of $\Sigma$ and $\langle\bar{\psi} \psi\rangle$ coincide again. The two limits of the gap equation give

$$
\begin{aligned}
& t=a g^{2} \Sigma^{2} \ln \frac{b}{\Sigma^{2}} \quad(m=0) \\
& m=a g^{2} \Sigma^{3} \ln \frac{b}{\Sigma^{2}} \quad(t=0)
\end{aligned}
$$

Up to logarithms the exponents have their gaussian values $\beta=1 / 2$ and $\delta=3$.

There are two universal quantities to be extracted from the susceptibility. In the critical region, in the chiral limit, the susceptibility scales as $\chi \approx C|t|^{-\gamma}$. The amplitude $C$ is different in the two phases and the ratio $C_{+} / C_{-}$is a universal quantity. Mean field theory predicts $C_{-} / C_{+}=2[21]$. In the symmetric phase it is easy to compute both $C_{-}$ and $\gamma$. Setting $\Sigma=0$ in eq.(2.4) and using the definition of the critical coupling, we get

$$
\chi^{-1}=1-g^{2} / g_{c}^{2}=(-t)
$$

which implies $\gamma=1, C_{-}=1$. In the broken phase the first two terms in eq.(2.4) cancel because of the gap equation. Combining the third term in eq.(2.4) with the gap equation (2.5), gives $\chi^{-1}-2 t \sim \Sigma^{2}$ and the expression for the susceptibility becomes

$$
\chi^{-1}=\left(2-\frac{2}{\ln \left(b / \Sigma^{2}\right)}\right) t
$$

Clearly, the exponent $\gamma=1$ is the same as in the symmetric phase. There are no logarithmic corrections to scaling for the susceptibility. The amplitude, however, receives corrections due to scaling violations so that

$$
\frac{C_{-}}{C_{+}}=2-\frac{2}{\ln \left(b / \Sigma^{2}\right)}
$$

The wavefunction renormalization constant is given by the $k^{2}$ term of the $\sigma$ propagator. Due to radiative corrections such a term is generated in the $1 / N$ expansion. The leading log contribution is given by

$$
Z^{-1}=2 g^{2} \int_{q} \frac{1}{\left(q^{2}+\Sigma^{2}\right)^{2}} \sim \ln \frac{1}{\Sigma^{2}}
$$

The anomalous dimension $\eta$, defined by $Z \sim \Sigma^{\eta}$, vanishes up to logarithms. 
There are basically two candidates for the correlation length in this model: the fermion and $\sigma$-masses. It is simple to verify that they both scale the same way. The mass of the $\sigma$ particle, for example, is obtained through the second moment $\chi$. Using eqs.(2.8) and (2.10) leads to $M^{2}=Z \chi^{-1} \sim \Sigma^{2}$ The scaling of the fermion mass, $\Sigma$, is obtained easily from the gap equation, (2.7a). It follows that $\nu=\beta$, and so $\nu=1 / 2$ with the same scaling violations as for the order parameter.

The specific heat is obtained from the effective action, eq.(2.2). Like the susceptibility, it too is characterized by two quantities, the exponent $\alpha$ and amplitude $A$. In the critical region its scaling is given by $C=A|t|^{-\alpha}$. The amplitudes are different in the two phases and their ratio $A_{-} / A_{+}$is universal [21]. Mean field theory predicts $\alpha=0, A_{-}=0$ and the specific heat undergoes a jump. Unlike other exponents, $\alpha$ can be either positive or negative. In four dimension logarithmic corrections affect the scaling of $C$. However, to leading order in $1 / N, A_{-}$remains zero. It is easy to see this since in the symmetric phase, $<\sigma>=0$, and the action is independent of $g$. Thus, $A_{-}=0$. In the broken phase, straightforward algebra leads to

$$
C \sim \frac{1}{\ln (1 / t)}
$$

Higher order corrections might change the power of the logarithm, but it remains in the denominator. For scalar theories there are several results on the amplitude ratio and critical index $\alpha$ ( $\epsilon$-expansion and large- $N)$ [22]. In the symmetric phase $A_{-}=O(1 / N)$, so it does not appear in leading order. The amplitude ratio, therefore, scales as $1 / N$. In four dimension, $\alpha=0$ as well, but with logarithmic corrections of the form $C \sim$

$1 /(\ln t)^{(N-4) /(N+8)}$. Thus, depending on $N$, the power of the logarithm can change it's sign and the specific heat can change its behavior from vanishing to divergent. The $\epsilon$ expansion studies indicate that this does not happen in Yukawa models [19].

\section{Triviality of the four-dimensional theory in the large- $N$ limit}

To establish the connection between scaling violations and triviality, we introduce the renormalized coupling. It is a dimensionless low-energy quantity that contains information about the non-gaussian character of the theory. It is conventionally defined as [23]

$$
g_{R}=-\frac{\chi^{(n l)}}{\chi^{2} \xi^{d}}
$$


where the nonlinear susceptibility $\chi^{(n l)}$ is the zero-momentum projection of the connected four-point function

$$
\chi^{(n l)}=\frac{\partial^{3} M}{\partial h^{3}}=\int_{123}<\sigma(0) \sigma(1) \sigma(2) \sigma(3)>_{c}
$$

The normalization factors, $\chi=\int_{x}<\sigma(0) \sigma(x)>_{c}$ and $\xi^{d}$, in eq.(3.1) take care of the four fields and the three integrations. In a gaussian theory all higher-point functions factorize, so $g_{R}$ vanishes. Using the hyperscaling hypothesis, this can be converted into,

$$
g_{R} \sim \xi^{(2 \Delta-\gamma-d \nu) / \nu}
$$

where $\Delta=\beta+\gamma$ is the gap exponent. Being dimensionless, $g_{R}$ should be independent of $\xi$ if $\xi$ is the only scale. Thus, the validity of hyperscaling requires that the exponent must vanish. It implies the relation, $2 \Delta-\gamma-d \nu=0$, between the critical indices. In general, it is known that the following inequality [24] holds

$$
2 \Delta \leq \gamma+d \nu
$$

The exponent in the expression for $g_{R}$ is always non-positive, so that violations of hyperscaling imply that the resulting theory is non-interacting. Above four dimension, the exponents are gaussian $(\gamma=1, \Delta=3 / 2, \nu=1 / 2)$. In this case, it is easy to verify the above inequality: $3 \leq 1+d / 2$, which amounts to $d \geq 4$. In four dimension there are logarithmic corrections to scaling that are believed to drive $g_{R}$ to zero. Scaling violations in any thermodynamic quantity propagate into the renormalized coupling and, according to eq.(3.4), these violations lead to triviality. Because this is a marginal case, it requires special treatment.

In order to make our point simple, we illustrate how logarithms appear in the effective actions of two solvable models: $\phi^{4}$ and $(\bar{\psi} \psi)^{2}$ theories both in the large- $N$ limit. The effective actions for the two models are [5,25]

$$
\begin{gathered}
V(M)=-\frac{1}{2} t \frac{M^{2}}{\ln (1 / M)}+\frac{\lambda}{4} \frac{M^{4}}{\ln (1 / M)} \\
V(\sigma)=-\frac{1}{2} t \sigma^{2}+\sigma^{4} \ln (1 / \sigma)
\end{gathered}
$$

This is the leading log contribution only. In the first example, it is clear how log-corrections lead to triviality. The logarithm can simply be thought of as coming from the running 
coupling - quantum corrections lead to the replacement $\lambda \rightarrow \lambda_{R}$. The vanishing of the renormalized coupling is then manifest from eq.(3.5a). In the case of fermions, eq.(3.5b), the details are completely different - the analogous reasoning would lead to an erroneous conclusion that the renormalized coupling increases in the infrared. This form is obtained from the effective action, $S_{\text {eff }}(\sigma)$, in eq.(2.3). It is easy to see how the logarithm in $(3.5 \mathrm{~b})$ appears in the numerator. For example, after taking the second derivative of $\chi^{-1}$, eq.(2.4), the last term generates a logarithmically divergent contribution which is the log-term in eq.(3.5b). The vanishing of the renormalized coupling here follows from the wave function renormalization constant $Z \sim 1 / \ln (1 / \sigma)[5]$.

In both cases the renormalized coupling is obtained through the nonlinear susceptibility. The correlation length is related to the susceptibility by $\xi^{2}=\chi / Z$. For magnets (i.e. scalar theories like $\phi^{4}$ ) the following relations hold,

$$
\begin{gathered}
\chi^{(n l)} \sim \chi^{4} \frac{\lambda}{\ln (1 / M)}, \quad Z=1 \\
g_{R} \sim Z^{2} \frac{\lambda}{\ln (1 / M)} \sim \frac{1}{\ln (1 / M)}
\end{gathered}
$$

so $g_{R}$ vanishes at a logarithmic rate.

For fermions, on the other hand, we have

$$
\begin{gathered}
\chi^{(n l)} \sim \chi^{4} \ln (1 / \sigma), \quad Z \sim \frac{1}{\ln (1 / \sigma)} \\
g_{R} \sim Z^{2} \ln (1 / \sigma) \sim \frac{1}{\ln (1 / \sigma)}
\end{gathered}
$$

and again $g_{R}$ vanishes at a logarithmic rate. In this context the following point should be made. The nonlinear susceptibility in the NJL model is a connected four-point function for the composite $\bar{\psi} \psi$ field. The free fermionic theory is not gaussian in $\bar{\psi} \psi$, so even in free field theory $g_{R}$, defined in this way, does not vanish. The fact that $g_{R} \rightarrow 0$ near the critical point indicates not only that the resulting theory is gaussian, but that it is also a purely bosonic theory. So, for the NJL model, the bare theory and its continuum limit bear no resemblance. This is quite different from what happens in the $\phi^{4}$ model where the resulting continuum limit has the same physical content as the bare theory but with vanishing coupling constant.

The important point in this comparison of the two models is the fact that the logarithms in the EOS appear in different places. This property is generic for the two models 
and persists beyond the $1 / N$ expansion. In order to emphasize this point, we consider the critical EOS for both models. They are obtained from the effective potential by simple differentiation. To make the connection with $\delta$, we take $t=0$. The critical EOS for the magnets is $[25]$

$$
h \sim \frac{M^{3}}{\log (1 / M)}
$$

This defines the exponent $\delta$. Because of the scaling violations, eq.(3.8) vanishes faster than a pure power. So the "effective" $\delta$ is bigger then its mean field value. It is easy to understand the correction to scaling in eq.(3.8). $1 / \delta$ is simply the logarthmic derivative of the order parameter $1 / \delta=\partial \ln M / \partial \ln h$. Since the magnetization saturates at strong fields, its slope vanishes in the large- $h$ limit. Thus, $1 / \delta$ decreases away from the $h=0$ limit. As was shown in ref.[20], such corrections to scaling can never occur in the case of the chiral transition.

For the NJL model the critical EOS reads,

$$
m \sim \sigma^{3} \log (1 / \sigma)
$$

Unlike scalar theories, the log's appear in the numerator - the right hand side in eq.(3.9) vanishes slower than the pure power and the "effective" $\delta$ is smaller than the (pure) mean field value. This is also easy to understand since at large mass $\langle\bar{\psi} \psi>\sim m$. Thus, away from the chiral limit $1 / \delta$ is driven to 1 . As pointed out in ref.[20] this difference in the position of the logarithm, eqs.(3.8-9) or, equivalently, the sign of the scaling violations is generic for the two models and is intimately tied to the fact that in NJL scalars are composite and in $\phi^{4}$ they are elementary.

The distinction between the two models proved to be useful in analyzing the lattice data. In particular, if the NJL model is simulated on a small lattice, then, for a fixed mass, the right hand side of eq.(3.9) will be smaller than it is in the thermodynamic limit because finite volume effects tend to restore the symmetry, and reduce the order parameter. If the infinite volume $\sigma$ is to be fitted to the data in the form $m \sim \sigma^{\delta^{\prime}}$, then the appropriate exponent $\delta^{\prime}$, which might fit the data, must be bigger than its actual value. This larger value of $\delta$ could also be mimicked by mean field scaling supplemented with the $\log$ in the denominator. Such data should be not be trusted since their behavior is qualitatively different from the behavior of the system in the thermodynamic limit. As the lattice size is increased, the effective $\delta$ would eventually fall below its mean field value 
suggesting the proximity of the thermodynamic limit. Such data, although not necessarily completely free of finite size distortions, do share the same qualtiative features with the physics of the infinite volume. So, for fermions the bound $\delta<3$ separates the data into two groups and tells us which one is more reliable. This is possible because finite size distortions and the approach to the true scaling law have the opposite tendencies. For scalar theories, on the other hand, the two have the same tendency. Nontrivial scalar theories have $\delta>3$, to begin with. For example, from eq.(3.8) it is apparent that in case of logarithmic corrections to mean field scaling, the finite size distortions which tend to reduce the magnetization can be masked by adjusting either the exponent $\delta$ or the strength of the logarithm so that the right hand side of the critical EOS assumes an appropriate magnitude compatible with the given $h$. In fact, strong finite size effects would force the fit with $\delta>3$ even if the scaling is mean field, so work on a larger lattice is necessary to either confirm or disprove this result. Thus, in scalar theories a careful finite size scaling analysis seems to be necessary to disentangle the log's from the finite size effects. In that sense, establishing triviality in $\phi^{4}$ is more delicate than it is in fermionic theories [6].

\section{Simulation results}

We carried out extensive simulations of the model described by Eq.(1) on $8^{4}$ and $16^{4}$ lattices. The small lattice simulations were done to monitor finite size effects. Of course, many more calculations on a variety of lattice sizes could be done to make our considerations on finite size effects more quantitative, but $8^{4}$ and $16^{4}$ simulations will prove adequate for our purposes. If Four Fermi models continue to play an ever increasing role in high energy theory, then more thorough studies would be needed.

Although there are some cases in which the finite size effects were large and curious as we shall see below, most of the $16^{4}$ simulations were relatively free from finite size problems for rather wide ranges of couplings and yielded precise continuum scaling laws. In fact, the logarithms of triviality will be seen clearly in our study of the Equation of State. There are several reasons for this success. First, the model can be simulated for zero quark mass. So we are free of the difficult issues of extrapolations to the chiral limit. Second, the discrete character of the symmetry breaking made the Hybrid Monte Carlo algorithm particularly effective and allowed us to run the simulations with a relatively large time step $(\mathrm{dt}=0.1)$ while still having a high acceptance rate (from 60 to 90 percent was typical). Third, we 
could dial the number of flavors large to compare to the $1 / N$ expansion (we chose $N_{f}=12$, as in our past work in three dimension [18]) which suppresses fluctuations enormously.

As discussed in the theory sections above, scalar systems and fermion systems are clearly distinguished by the positions of the scale breaking logarithms in their critical behaviors. One of the more interesting features we discovered here is that those distinct behaviors can be blurred by finite size effects. Comparison between $16^{4}$ result and $8^{4}$ clearly illustrates such effects.

The lattice action, the Hybrid Monte Carlo algorithm and other technical details of the simulations follow ref[18] in detail. The interested computational physicist should consult that reference for details. In our discussion and Tables, we shall add any technical information which was special to working in four dimension.

For clarity, we shall organize our lattice simulation results into various subtopics, following the theoretical discussion above.

\section{Equation of State}

In Table I we have collected the $16^{4}$ data for the average action, its specific heat, the vacuum expectation value of the $\sigma$ field, and its susceptibility $\chi$. We also tabulate there the statistics of each measurement and the statistical errors on each quantity. The errors were calculated with considerable care using the usual binning techniques, because we are interested in high quality fits to distinguish different functional forms in the model's critical behavior. Note that in the immediate vicinity of the critical point, we accumulated particularly high statistics (1/3 of a million sweeps) to combat critical slowing down.

We first considered the order parameter's dependence on coupling in the broken symmetry phase. The logarithmic fit,

$$
\beta-\beta_{c}=a \sigma^{2}(\ln (1 / \sigma)+b)^{p}
$$

was tried for the twelve data points in the coupling range $\beta=.54-.595$. A very good fit was found (confidence level=70 percent) and the parameters were determined: $\beta_{c}=.6044(1)$, $a=2.25(2), b=1.16(5)$, and $p=1.041(12)$. The data and fit are shown in Fig. 1a.

Perhaps the most interesting feature of this result is the fact that the power of the logarithm in Eq.(4.1) is determined with such precision and confidence. The fitting routine (standard least square method) searched for the best value of the power and found 1.041(12). Note that the fit does not just accommodate the immediate vicinity of the 
critical point : the parameter ' $b$ ' in Eq.(4.1) which sets the scale of the logarithm allows us to fit both the strong coupling region (where $\sigma$ is large and mean field scaling should apply) and the critical region (where fluctuations causes the logarithmic correction to the mean field behavior of the model).

It is interesting to plot the data in a slightly different way to show the importance of the logarithm more visually. Consider Fig. 2a where we plot the data as $\left(\beta-\beta_{c}\right) / \sigma^{2}$ vs. $\ln (1 / \sigma)$ for the choice $\beta_{c}=.6044$. The numerical significance of the logarithm and the fact that its power is very close to 1.00 are apparent.

We also attempted equation of state fits which are free of logarithms. For example, if the theory had pure power law singularities, then the form,

$$
\beta_{c}-\beta=a \sigma^{1 / \beta_{\text {mag }}}+b \sigma^{2}
$$

should apply in the broken phase. When a least square fit of this form is attempted, the iterative procedure ( the search for the best confidence level ) does not converge, although high confidence levels are found for fixed fitting parameters. The fitting program finds that it can always increase the confidence level by increasing ' $a$ ' positively, while increasing 'b' negatively and driving $1 / \beta_{\text {mag }}$ to 2 from above. Such a tendency is reminiscent of the $\epsilon$-expansion where the limit of $d=4$ is marked by the fact that power law scaling is converted into logarithms through the formula $\ln x=\lim _{\epsilon \rightarrow 0}\left(x^{\epsilon}-1\right) / \epsilon$. The behavior of the fit is compatiable with the weaker logarithmic dependence on $\sigma$ found above. Our data is sufficiently accurate and it extends over a wide enough range of $\beta$ to favor the logarithmic equation of state over the pure power law formula.

In Table II we collect our $8^{4}$ measurements which parallel the $16^{4}$ measurements presented in Table I. First consider the equation of state in the broken phase. Fits of the form Eq (4.1) and (4.2) which should have worked in both the strong coupling and critical regions were unsuccessful ( the confidence levels were infinitesimal ). However, if we took a subset of the data in the critical region alone reasonable fits could, of course, be made. For example, a pure power law fit such as,

$$
\sigma=a\left(\beta_{c}-\beta\right)^{p}-b
$$

could be made over the relatively narrow region from $\beta=.57$ to .595 with a fair confidence level (20 percent) and results: $\beta_{c}=.607, a=1.56(9), p=.54(3)$, and $b=.002(13)$. The 
fit is shown in Fig. 1b. Note that the fit is consistent with mean field theory, unadorned with logarithms.

To gain more insight into the problems with $8^{4}$ simulation data, let us plot it as we did the $16^{4}$ data in Fig. 2a. The result is shown in Fig. 2b. Comparing with Fig. 2a, it appears that the $8^{4}$ data suffers from finite size effects as $\beta$ approaches the critical point and there are only 'hints' of the true logarithmic corrections in Fig. 2b. This plot shows clearly that it would not be sensible to 'blindly' fit $8^{4}$ data to any continuum model scaling law, even though the $16^{4}$ data shows continuum behavior over the same range of couplings.

\section{Susceptibility}

The measurements of the order parameter $\sigma$ allow us to compute its variance and construct the model's longitudinal susceptibility $\chi$. The data is given in Table I. First consider the broken symmetry side of the transition and a determination of the critical index $\gamma$ assuming a pure power law singularity. For $\beta$ ranging from .54 through .595, we fit the susceptibility data with

$$
\chi^{-1}=a\left(\beta_{c}-\beta\right)^{\gamma}+c
$$

We are particularly interested in fits which produce an estimate of the parameter 'c' consistent with zero, indicating that $\beta_{c}$ is a critical point. The least square routine gives: $a=9.14(1.12), \gamma=0.967(45), c=0.002(10)$ for the choice $\beta_{c}=.6028$. If trial values of $\beta_{c}$ are chosen larger than .6045 or smaller than .6022 , then the parameter ' $c$ ' is pushed significantly away from zero. However, for all such choices, $\gamma$ is found to be .967(45). So this result is very robust given a power law hypothesis. The data and the fit are shown in Fig. 3a. the confidence level of the fit is 63 percent (chi-squared $=7.13$ with nine degrees of freedom).

As discussed in the theoretical sections above, there are weak logarithmic corrections to power law scaling of the susceptibility in the broken phase. So, it is interesting to try fits of the form,

$$
\chi^{-1}=a\left(2-\frac{1}{\ln (1 / \sigma)+1.16}\right)\left(\beta_{c}-\beta\right)^{\gamma}+c
$$

where the scale in the logarithm (i.e. 1.16) has been taken from the fit Eq.(4.1). Again, very good fits are found. Requiring that ' $c$ ' be compatiable with zero, produces the prediction $\beta_{c}=.6040(8), \gamma=1.054(47)$, and $a=7.41(95)$. Although $\beta_{c}$ is not predicted as accurately 
as in the Equation of State fits, the critical index $\gamma$ is again found for all choices of $\beta_{c}$ to be 1.054(47). The confidence levels of these fits are typically 55 percent. Clearly the logarithm in Eq.(4.5) is not very significant numerically due to the presence of the substantial additive term 2. If we had a wider range of $\sigma$ values, the logarithm would play a greater role. The logarithms of triviality are much more easily exposed in other observables of this model.

Now consider the susceptibility on the symmetric side of the transition. No logarithms are expected in the scaling law here, so we try a fit of the form Eq.(4.4). We find excellent fits (confidence levels of 99 percent) with : $a=5.62(1.65), \beta_{c}=.6024(7)$, and $\gamma=1.0(1)$. Again, $\gamma$ is compatiable with unity, as expected theoretically at large N. The fit is shown in Fig. 3a.

For the $8^{4}$ lattice, we accummulated susceptibility data in both the strong and weak coupling phases, and we attempted fits of the form Eq.(4.5) that were successful on the $16^{4}$ lattice. To avoid vacuum tunnelling we did not study couplings too close to the critical point. The ranges of $\beta=.57-.595$ and .62-.655 satisfied this criterion. The data and power law fits are shown in Fig. 3b. In the broken phase the fit gave a critical index $\gamma$ $=.97(12)$, compatiable with theoretical expectations. However, the confidence level of the fit is barely acceptible (9.6 percent corresponding to a chi-squared of 6.4 with 3 degrees of freedom), and the other parameters in the fit were $\mathrm{a}=11.3(3.7)$ and $\mathrm{b}=-.029(29)$ for a critical coupling of $\beta_{c}=.6066$. Note that the crude strong coupling data shown in the figure at $\beta=.54, .55$, and .56 were not used in the fit. The weak coupling data and its fit are also shown in the figure. The confidence level was very good (93.9 percent) and the fit predicted the critical index $\gamma=1.21(10)$ with $\mathrm{a}=9.8(2.8)$ and $\mathrm{b}=.025(8)$ for $\beta_{c}=$ .6066. So the fitted $\gamma$ is two standard deviations from the theoretical prediction. Since the $16^{4}$ fits work beautifully with $\gamma=1.05(5)$, this discrepancy is most probably a finite size effect.

In summary, the $8^{4}$ susceptibility study is not quantitatively reliable, in contrast to the $16^{4}$ results which are very close to the continuum model predictions.

\section{Equation of State at Criticality}

Another feature of the theory which displays the logarithm of triviality in an accessible expression is the equation of state at criticality. In Sec.3 above we contrasted the behavior of scalar vs. fermion models in Eq.(3.8) and Eq. (3.9). To distinguish the two behaviors we must simulate the model at $\beta_{c}$ in the presence of explicit symmetry breaking. We 
anticipate that this work will be subject to large finite size effects and will not be as quantitative as our other measurements which avoided all the pitfalls of the critical point. We shall see that our results will favor Eq.(3.9) over Eq.(3.8), and a comparison with $8^{4}$ measurements will expose some particularly interesting finite size effects.

We ran simulations at nonzero fermion mass $\mathrm{m}$ at $\beta=.6034$. The results are compiled in Table III. It would be interesting to repeat this work at other estimates of $\beta_{c}$, but high statistics are needed since we are working very close to criticality and statistical fluctuations are large. We begin by plotting the data in Fig. $4 \mathrm{a}\left(m / \sigma^{3}\right.$ vs. $\left.\ln (1 / \sigma)\right)$ in order to discriminate between scalar and fermion behavior. The figure shows a 'window' in $\sigma$ where the logarithm of triviality is in the numerator of Eq.(3.9). However, it appears that only a small range of $\sigma$ is relevant : for large $\mathrm{m}$ (greater than .015) the scaling form of Eq.(3.9) probably does not apply, and for small m (less than .002) finite size effects are probably distorting the $16^{4}$ data. We will gain a better perspective when the $8^{4}$ data is plotted. The solid line showing logarithmic behavior is just meant to guide the eye systematic fitting procedures are not appropriate here.

We also measured the susceptibility at criticality. It follows from eq.(2.4) that,

$$
\chi \sim \frac{1}{\sigma^{2} \ln (1 / \sigma)}
$$

so a plot of $\chi^{-1} / \sigma^{2}$ vs. $\ln (1 / \sigma)$ should be linear with a positive slope. Fig.5 shows that the data is compatiable with this expectation, but the data suffers from large error bars which make precise predictions meaningless here.

The $8^{4}$ data in this case will be subject to much larger and interesting finite size effects. The data is shown in Table IV for an estimate of $\beta_{c}=.6066$ which follows from the equation of state and susceptibilities. Masses ranging from .001 through .020 were simulated, and the results are plotted in Fig. 4b in the form suggested by Eq.(3.9). We find that the plot of $m / \sigma^{3}$ vs. $\ln (1 . / \sigma)$ has a negative slope! This should be compared with Fig.4a, where an identical plot was made on the larger lattice $\left(16^{4}\right)$. The two plots differ qualitatively. In fact, if we plot $\sigma^{3} / m$ vs. $\ln (1 . / \sigma)$ as suggested by Eq.(3.8), the scalar field prediction, then a reasonable plot shown in Fig. 6 follows. This bizarre situation shows that finite size effects distort the true behavior of Eq.(3.9), as found on the $16^{4}$ lattice, into something compatiable with the scalar field expectation. This should be a severe warning for other simulation studies : finite size effects can even obscure the fundamental fermion and/or scalar character of the underlying theory. 


\section{The Average Action and the Specific Heat}

Since accurate measurements of the average action and its associated specific heat are relatively easy, let us discuss them briefly. The data is taken from Table I, and the average action is plotted vs. $\beta$ in Fig.7a. We see a clear change in slope near $\beta \sim .60$. This effect is clearer in the specific heat which is plotted in Fig.8a. As discussed in the theory sections above, mean field theory predicts a jump in the specific heat at the critical point. Possible curvature and logarithms in such plots are not visible given the error bars in the figures and the fact that we could not simulate the model closer to $\beta_{c}$ due to finite size effects such as vacuum tunnelling. Nonetheless, the (chiral) phase transition is apparent in the plots.

The $8^{4}$ data and plots for the average action and its associated specific heat are given in the table II and Fig. $7 \mathrm{~b}$ and $8 \mathrm{~b}$. These results are qualitatively similar to the $16^{4}$ results discussed above. They were not useful in subtle studies of logarithms. The general agreement between the data from the two lattice sizes is not unexpected since these quantities are dominated by ultraviolet fluctuations.

\section{Discussion and conclusions}

The main conclusions of our study are the following. Logarithmic corrections to mean field scaling in the EOS are properly predicted by the large- $N$ expansion. Our data demonstrate this with a high degree of confidence. In fact, when we tried to impose the only remaining possibility, power law scaling in the form of eq.(4.2), not only did the fit fail, but it did so in an illuminating fashion - the parameters of the fit flowed toward a polynomial generator for the logarithm itself. A pure mean field form worked only if a restricted range of data were used.

We have seen that finite size effects appear in a peculiar fashion and we explained the criteria that can detect the finite volume distortions when they are present. These criteria are of broader significance since they originate from studies of the simplest of the models in the class - NJL with discrete chiral symmetry. Other models with dynamical symmetry breaking, like NJL with continuous chiral symmetry or massless fermionic $Q E D$, are subject to more severe finite volume effects because of their Goldstone bosons. In that context, the most striking feature of our study can be summarized in the comparison of the figures $4 \mathrm{a}$ and $4 \mathrm{~b}$. They depict identical plots on two different lattice sizes and, because of the way the plots are designed, the difference is seen as qualitative. Theoretical input, 
layed out in ref[20] and summarized in Sec.3, would have enabled us to discard the $8^{4}$ data even if we had had no access to the $16^{4}$ lattices. While in some instances, the $8^{4}$ data were not severely distorted (e.g. Fig.3b), in other instances, they were completely useless even as a guide for qualitative behavior. In fact, the logarithms and finite size effects in these cases interfere with each other and the data is misleading. Thus, on a small lattice, some fits would favor the logarithms in places where they simply can not appear.

As it turned out, establishing the log's in the NJL model was easier than the corresponding task in $\phi^{4}$ theory. It was not so crucial to have terribly high statistics as long as the lattice size was sufficiently large. Our preliminary results obtained with lower statistics did not differ considerably from the final ones. This advantage could be correlated with our control over the quality of data and the magnitude of the finite size effects through the bound on the exponent $\delta$.

Acknowledgement This work is supported by NSF-PHY 92-00148. S. Kim is supported by the U. S. Department of Energy, Contract No. W-31-109-ENG-38. 


\section{References}

[1] Y. Nambu and G. Jona-Lasinio, Phys. Rev. 122, 345 (1961).

[2] D. Lurie and A. Macfarlane, Phys. Rev. 136, Bb16 (1964); K. Tamvakis and G. Guralnik, Nucl. Phys. B146, 224 (1978); A. Hasenfratz, et al., Nucl. Phys. B365, 79 (1991); J. Zinn-Justin, Nucl. Phys. B367, 105 (1991).

[3] K. Wilson, Phys. Rev. D7, 2911 (1973).

[4] K. Gawedzki and A. Kupianen, Phys. Rev. Lett. 55, 363 (1985); B. Rosenstein, B. Warr and S. Park, Phys. Rev. Lett. 62, 1433 (1989); C. de Calan, P. Faria da Veiga, J. Magnen and R. Sénéor, Phys. Rev. Lett. 66, 3233 (1991).

[5] T. Eguchi, Phys. Rev. D17, 611 (1978); K.-I. Shizuya, Phys. Rev. D21, 2327 (1980).

[6] M. Aizenman, Comm. Math. Phys. 86, 1 (1982); C. Aragão de Carvalho, S. Caracciolo and J. Fröhlich, Nuc. Phys. B215[FS7], 209 (1983); M. Lüscher and P. Weisz, Nuc. Phys. B290[FS20], 25 (1987); R. Kenna and C. Lang, Nuc. Phys. B393, 461 (1993).

[7] A. Casher, Phys. Lett. 83B, 395 (1979).

[8] S. K. Ma, in Phase Transitions and Critical Phenomena Vol.6, eds. C. Domb and M. Green (Academic Press, London, 1976).

[9] K. Wilson and J. Kogut, Phys. Rep. 12C, 75 (1974).

[10] D. Gross and A. Neveu, Phys. Rev. D10, 3235 (1974).

[11] B. Rosenstein, B. Warr and S. Park, Phys. Rep. 205, 497 (1991).

[12] S. Hands, A. Kocić and J. B. Kogut, Phys. Lett. B273 (1991) 111.

[13] A. Dhar, R. Shankar and S. Wadia, Phys. Rev. D31, 3256 (1985).

[14] T. Hatsuda and T. Kunihiro, Tsukuba preprint, UTHEP-270.

[15] For a review, see T. Appelquist, Yale preprint, YCTP-P23-91.

[16] Y. Nambu, in New Trends in Physics, proceedings of the XI International Symposium on Elementary Particle Physics, Kazimierz, Poland, 1988, edited by Z. Ajduk S. Pokorski and A. Trautman (World Scientific, Singapore, 1989); V. Miransky, M. Tanabashi and K. Yamawaki, Mod. Phys. Lett. A4, 1043 (1989); W. Bardeen, C. Hill and M. Lindner, Phys. Rev. D41, 1647 (1990).

[17] K. Bitar and P. Vranas, FSU preprints, FSU-SCRI-93-127 and FSU-SCRI-93-130.

[18] S. Hands, A. Kocić and J. B. Kogut, Ann. Phys. 224, 29 (1993).

[19] L. Kärkkäinen, R. Lacaze, P. Lacock and B. Petersson, Saclay preprint, SPhT 93/053.

[20] A. Kocić and J. Kogut, Illinois preprint, ILL-(TH)-93-21.

[21] See, for example, C. Itzykson and J.-M. Drouffe, Statistical Field Theory (Cambridge 
University Press, 1989); V. Privman, P.C. Hohenberg and A. Aharony, in Phase Transitions and Critical Phenomena Vol.14, eds. C. Domb and J.L. Lebowitz (Academic Press, London, 1991).

[22] E. Brezin, J.-C. Le Guillou and J. Zinn-Justin, Phys. Lett. 47A (1974) 285; R. Abe and S. Hikami, Prog. Theor. Phys. 54 (1975) 1693.

[23] B. Freedman and G. A. Baker Jr, J. Phys. A15 (1982) L715.

[24] R. Schrader, Phys. Rev. B14 (1976) 172; B. D. Josephson, Proc. Phys. Soc. 92 (1967) 269, 276.

[25] E. Brezin, J.-C. Le Guillou and J. Zinn-Justin, in Phase Transitions and Critical Phenomena Vol.6, eds. C. Domb and M. Green (Academic Press, London, 1976). 


\section{Figure captions}

1a. The EOS fit Eq.(4.1) to the $16^{4}$ data.

1b. The EOS fit Eq.(4.3) to the $8^{4}$ simulation data.

2a. Same data as in Fig.1a fit to the logarithmic EOS Eq.(2.7a).

2b. $8^{4}$ data plotted following Fig.2a.

3a. Susceptibility data on the $16^{4}$ lattice and power law fits following Eq.(4.4).

3b. Susceptibility data and power law fits on $8^{4}$ lattice following Fig.3a.

4a. $16^{4}$ simulation data at criticality plotted following Eq.(3.9).

4b. $8^{4}$ simulation data at criticality plotted following Eq.(3.9). Note the qualitatively different behavior from $4 \mathrm{a}$.

5. Susceptibility data at criticality plotted as in Eq.(4.6).

6. $8^{4}$ simulation data at criticality plotted following scalar field theory. Finite size effects have distorted the data dramatically.

7a. Average action plotted vs. coupling on the $16^{4}$ lattice.

7b. $8^{4}$ analog of Fig.7a.

8a. Specific heat associated with Fig.7a.

8b. $8^{4}$ analog of Fig. 8 a. 


\section{Table I}

Final compilation $16^{4}$

\begin{tabular}{|c|c|c|c|c|c|}
\hline$\beta$ & $S$ & C & $\sigma$ & $x$ & sweeps $\left(\times 10^{4}\right)$ \\
\hline 0.30 & $4.8173(1)$ & $3.8(1)$ & $1.2123(1)$ & $0.50(1)$ & 2 \\
\hline 0.35 & $4.5661(3)$ & $3.6(2)$ & $1.0084(1)$ & $0.47(2)$ & 2 \\
\hline 0.40 & $4.3255(1)$ & $3.44(8)$ & $0.82828(5)$ & $0.55(1)$ & 3 \\
\hline 0.45 & $4.0967(4)$ & $3.438(4)$ & $0.6613(2)$ & $0.697(6)$ & 3 \\
\hline 0.50 & $3.8820(4)$ & $3.11(15)$ & $0.4974(2)$ & $0.988(41)$ & 3 \\
\hline 0.54 & $3.7233(3)$ & $3.01(6)$ & $0.3592(1)$ & $1.58(5)$ & 5 \\
\hline 0.545 & $3.7047(2)$ & $3.01(6)$ & $0.3408(1)$ & $1.71(4)$ & 5 \\
\hline 0.55 & $3.6864(2)$ & $3.01(6)$ & $0.3220(1)$ & $1.90(7)$ & 5 \\
\hline 0.555 & $3.6681(1)$ & $2.84(7)$ & $0.3025(1)$ & $2.03(7)$ & 6 \\
\hline 0.56 & $3.6504(1)$ & $2.80(7)$ & $0.2826(1)$ & $2.24(8)$ & 6 \\
\hline 0.565 & $3.6329(2)$ & $2.79(4)$ & $0.2619(2)$ & $2.56(4)$ & 9 \\
\hline 0.57 & $3.6159(1)$ & $2.81(6)$ & $0.2403(2)$ & $3.03(8)$ & 11 \\
\hline 0.575 & $3.5995(2)$ & $2.79(7)$ & $0.2179(2)$ & $3.61(10)$ & 10 \\
\hline 0.58 & $3.5839(2)$ & $2.73(6)$ & $0.1945(2)$ & $4.29(12)$ & 13 \\
\hline 0.585 & $3.5688(1)$ & $2.59(5)$ & $0.1693(2)$ & $5.26(11)$ & 22 \\
\hline 0.59 & $3.5544(2)$ & $2.55(4)$ & $0.1415(3)$ & $7.18(18)$ & 20 \\
\hline 0.595 & $3.5408(2)$ & $2.66(13)$ & $0.1091(4)$ & $12.07(37)$ & 32 \\
\hline 0.61 & $3.5200(1)$ & $1.002(8)$ & - & $24.59(1.76)$ & 13 \\
\hline 0.615 & $3.5194(1)$ & $0.971(12)$ & - & $14.33(96)$ & 10 \\
\hline 0.62 & $3.51901(1)$ & $0.968(11)$ & - & $10.40(65)$ & 8 \\
\hline 0.625 & $3.5186(1)$ & $0.972(5)$ & - & $8.02(37)$ & 9 \\
\hline 0.63 & $3.5184(1)$ & $0.978(8)$ & - & $6.70(23)$ & 10 \\
\hline 0.635 & $3.5181(1)$ & $0.985(11)$ & - & $5.61(18)$ & 11 \\
\hline 0.64 & $3.5178(1)$ & $0.996(10)$ & - & $4.93(17)$ & 11 \\
\hline 0.645 & $3.5176(1)$ & $1.022(12)$ & - & $4.18(17)$ & 9 \\
\hline 0.65 & $3.5173(1)$ & $1.023(12)$ & - & $3.82(13)$ & 9 \\
\hline
\end{tabular}




\section{Table II}

Final compilation $8^{4}$

\begin{tabular}{|c|c|c|c|c|c|}
\hline$\beta$ & $S$ & $C$ & $\sigma$ & $x$ & sweeps $\left(\times 10^{4}\right)$ \\
\hline 0.54 & $3.7385(4)$ & $2.97(1)$ & $0.3724(7)$ & $1.42(5)$ & 5 \\
\hline 0.55 & $3.7036(4)$ & $2.97(1)$ & $0.3384(7)$ & $1.65(5)$ & 5 \\
\hline 0.56 & $3.6699(6)$ & $2.88(9)$ & $0.3030(8)$ & $1.97(8)$ & 5 \\
\hline 0.57 & $3.6367(2)$ & $2.75(3)$ & $0.2650(2)$ & $2.38(3)$ & 30 \\
\hline 0.575 & $3.6206(2)$ & $2.76(4)$ & $0.2448(2)$ & $2.78(4)$ & 30 \\
\hline 0.58 & $3.6052(3)$ & $2.69(3)$ & $0.2242(3)$ & $3.23(5)$ & 30 \\
\hline 0.585 & $3.5890(2)$ & $2.71(3)$ & $0.2004(3)$ & $4.30(12)$ & 30 \\
\hline 0.59 & $3.5739(4)$ & $2.69(5)$ & $0.1750(8)$ & $5.93(29)$ & 30 \\
\hline 0.595 & $3.5584(6)$ & $2.57(2)$ & $0.1447(13)$ & $8.37(9)$ & 30 \\
\hline 0.62 & $3.5234(1)$ & $1.060(6)$ & - & $12.82(19)$ & 30 \\
\hline 0.625 & $3.5220(1)$ & $0.986(8)$ & - & $9.92(16)$ & 30 \\
\hline 0.63 & $3.5208(1)$ & $0.942(7)$ & - & $7.83(19)$ & 30 \\
\hline 0.635 & $3.5200(1)$ & $0.942(32)$ & - & $6.42(11)$ & 30 \\
\hline 0.64 & $3.5193(1)$ & $0.932(34)$ & - & $5.40(7)$ & 30 \\
\hline 0.645 & $3.5188(1)$ & $0.886(3)$ & - & $4.66(7)$ & 9 \\
\hline 0.65 & $3.5184(1)$ & $0.872(3)$ & - & $4.14(10)$ & 30 \\
\hline 0.655 & $3.5180(1)$ & $0.866(4)$ & - & $3.71(10)$ & 30 \\
\hline
\end{tabular}


Table III

Final Compilation at Criticality $\left(\beta_{c} \approx 0.6034,16^{4}\right)$

m

$\sigma$

0.001

0.002

0.003

0.004

0.005

0.010

0.015

0.020
$0.0962(3)$

$0.1212(2)$

$0.1389(2)$

$0.1532(2)$

$0.1653(2)$

$0.2093(3)$

$0.2401(1)$

$0.2644(1)$ $\chi$

$8.87(30)$

$5.87(4)$

$4.58(13)$

$3.68(9)$

$3.20(8)$

$2.11(2)$

$1.68(7)$

$1.40(2)$ sweeps $\left(\times 10^{4}\right)$

22

15

16

16

12

5

5

5 
Table IV

Final Compilation at Criticality $\left(\beta_{c} \approx 0.6055,8^{4}\right)$

\begin{tabular}{|c|c|c|c|}
\hline$m$ & $\sigma$ & $x$ & sweeps $\left(\times 10^{4}\right)$ \\
\hline 0.001 & $0.1007(9)$ & $16.3(8)$ & 20 \\
\hline 0.002 & $0.1360(7)$ & $6.9(6)$ & 20 \\
\hline 0.003 & $0.1559(6)$ & $4.3(5)$ & 20 \\
\hline 0.004 & $0.1698(5)$ & $3.4(4)$ & 20 \\
\hline 0.005 & $0.1808(5)$ & $3.1(3)$ & 20 \\
\hline 0.006 & $0.1905(4)$ & $2.7(2)$ & 20 \\
\hline 0.007 & $0.1998(4)$ & $2.4(2)$ & 20 \\
\hline 0.008 & $0.2076(3)$ & $2.2(2)$ & 20 \\
\hline 0.009 & $0.2147(3)$ & $2.0(2)$ & 20 \\
\hline 0.010 & $0.2218(3)$ & $1.9(2)$ & 20 \\
\hline 0.011 & $0.2278(2)$ & $1.9(2)$ & 30 \\
\hline 0.012 & $0.2341(3)$ & $1.7(1)$ & 30 \\
\hline 0.013 & $0.2398(2)$ & $1.6(1)$ & 30 \\
\hline 0.014 & $0.2457(4)$ & $1.5(1)$ & 30 \\
\hline 0.015 & $0.2506(4)$ & $1.4(1)$ & 30 \\
\hline 0.016 & $0.2555(3)$ & $1.4(1)$ & 20 \\
\hline 0.017 & $0.2594(2)$ & $1.3(1)$ & 20 \\
\hline 0.018 & $0.2639(2)$ & $1.3(1)$ & 20 \\
\hline 0.019 & $0.2683(2)$ & $1.2(1)$ & 20 \\
\hline 0.020 & $0.2726(2)$ & $1.2(1)$ & 20 \\
\hline
\end{tabular}




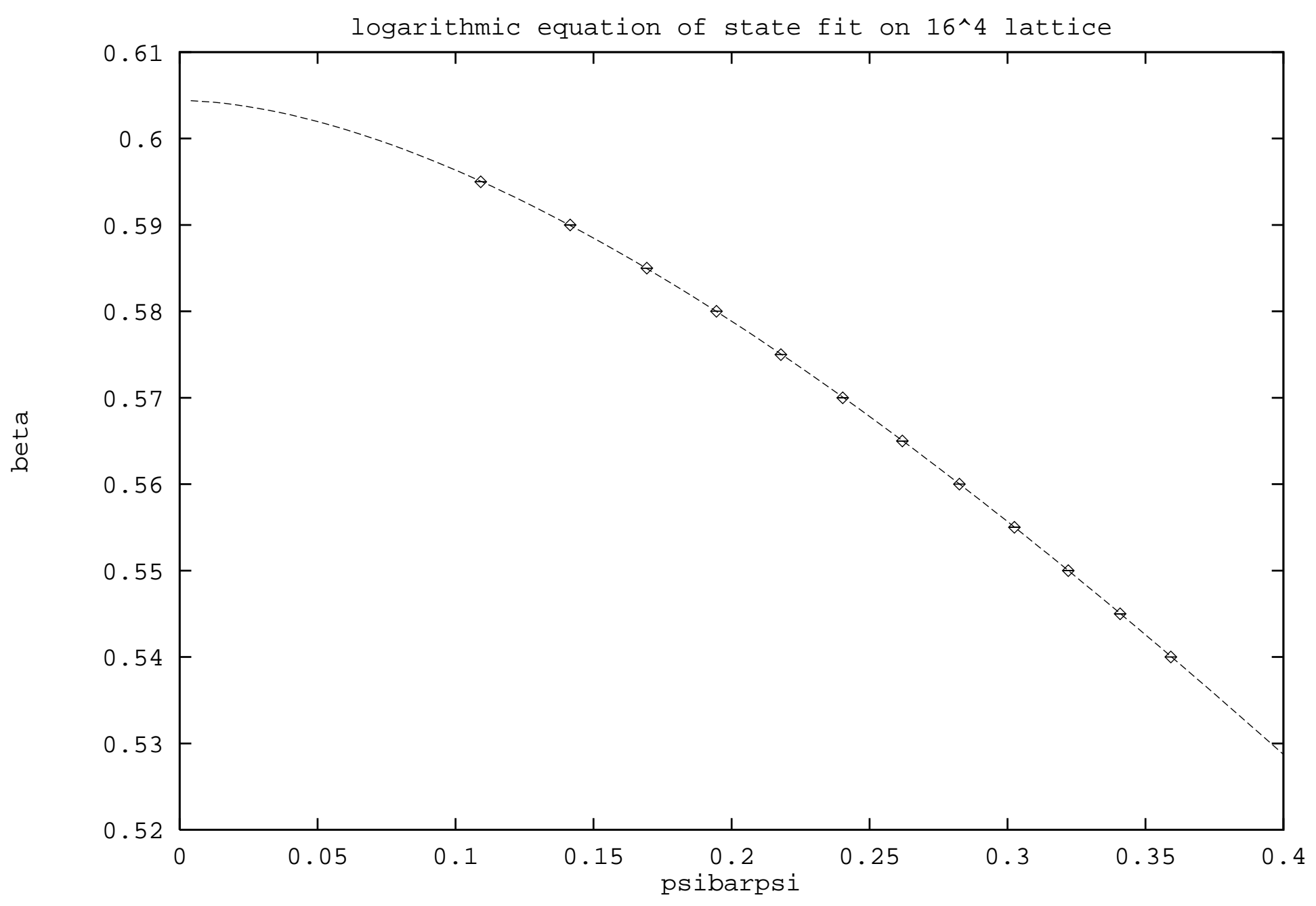









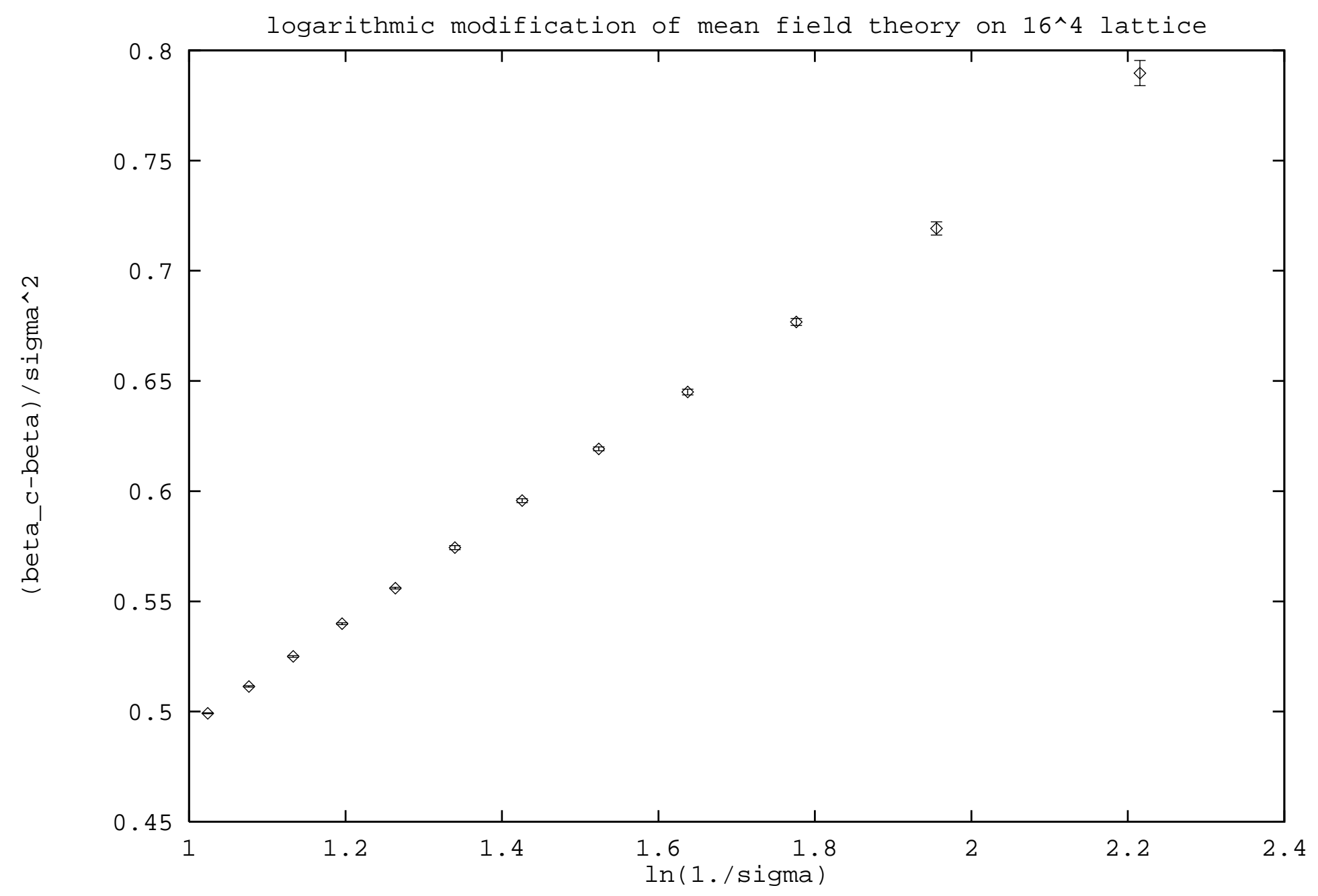




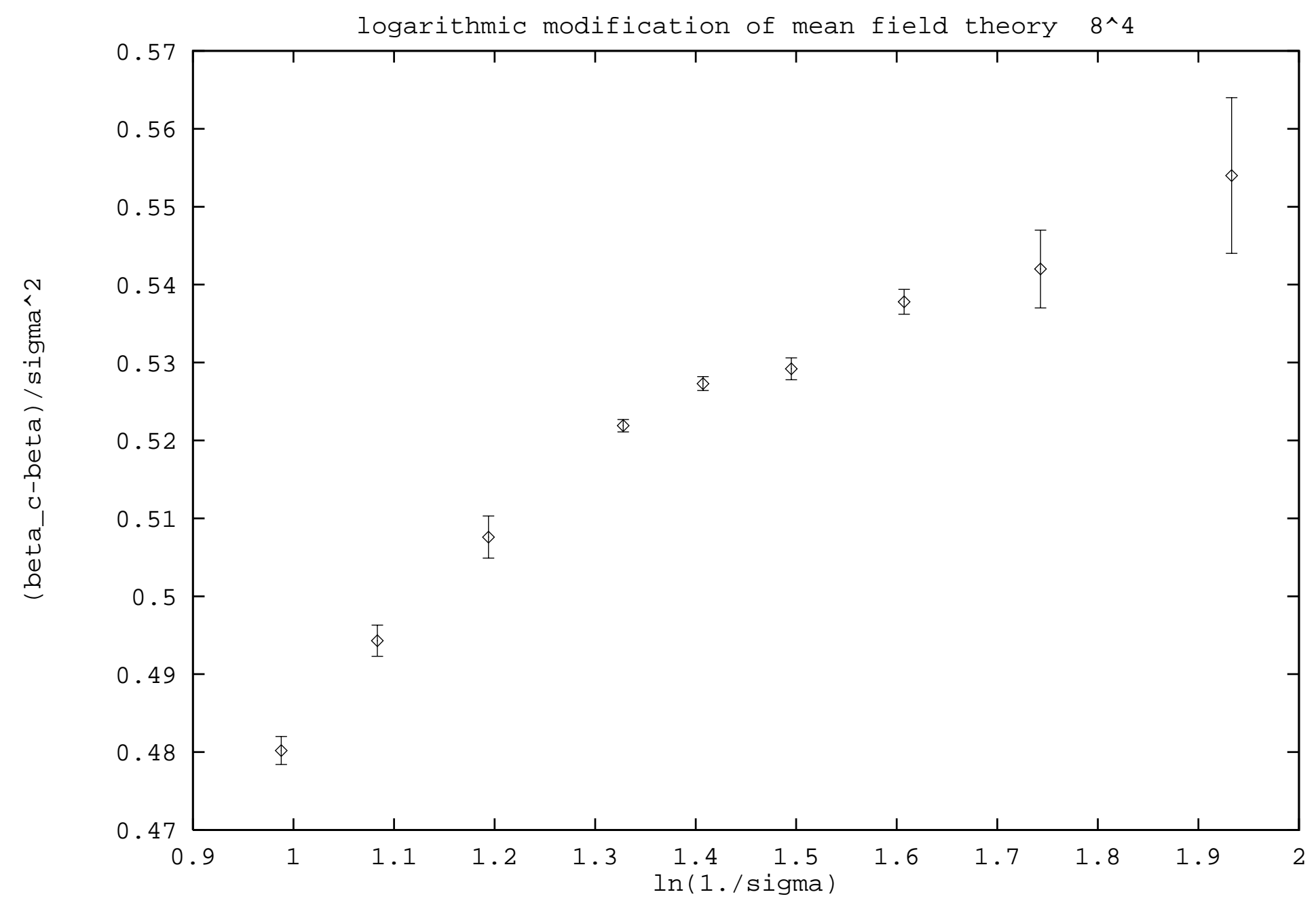




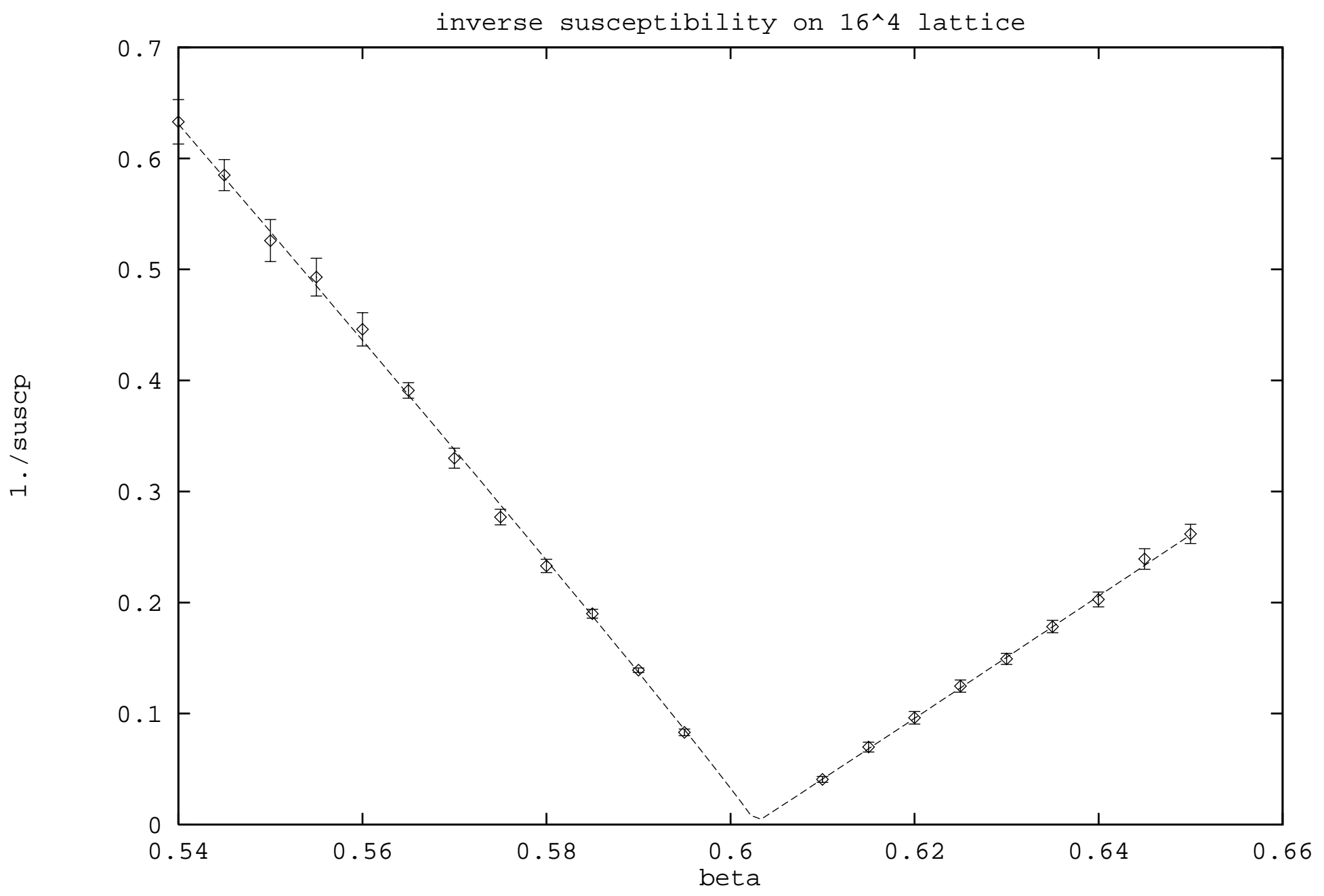














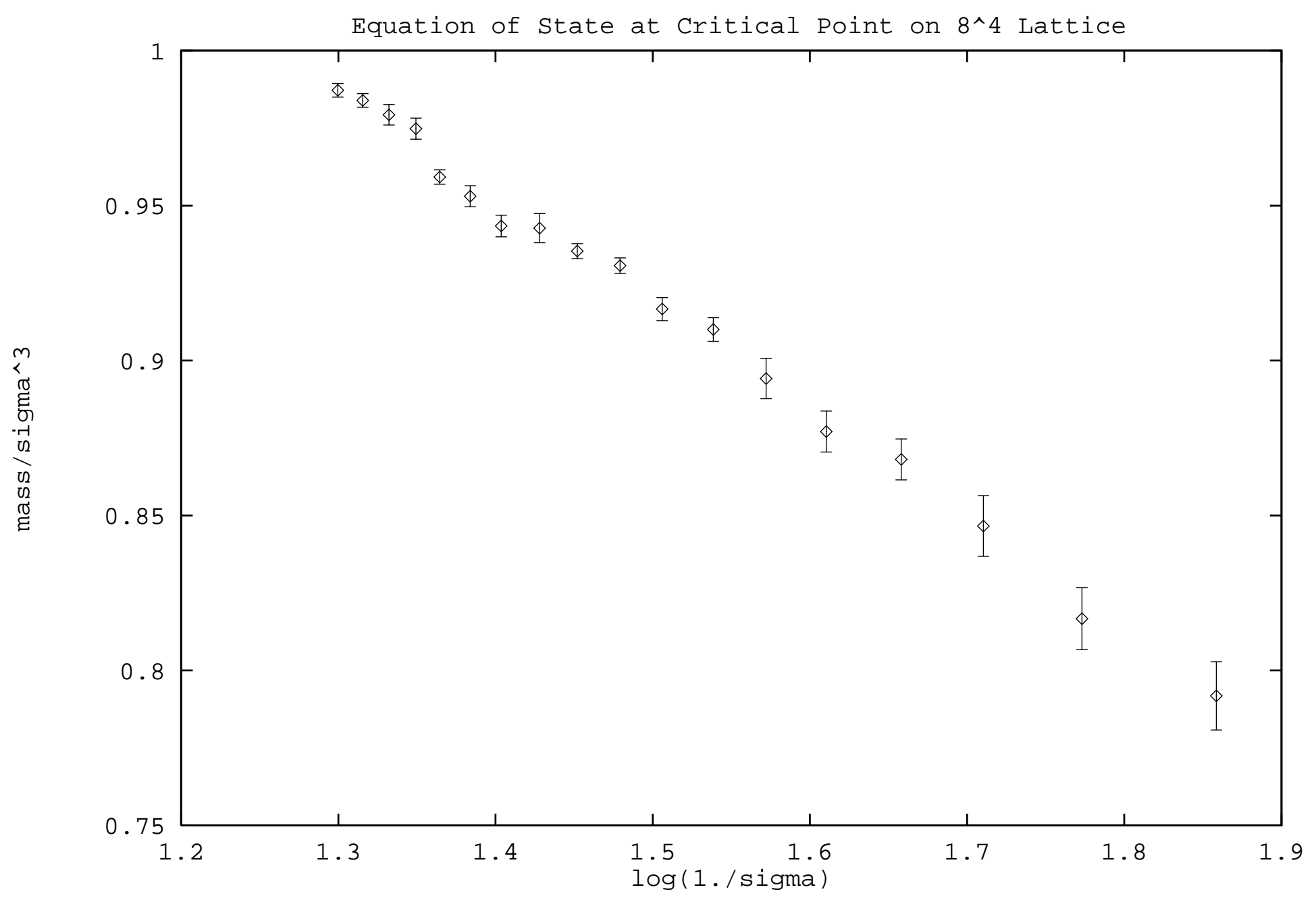




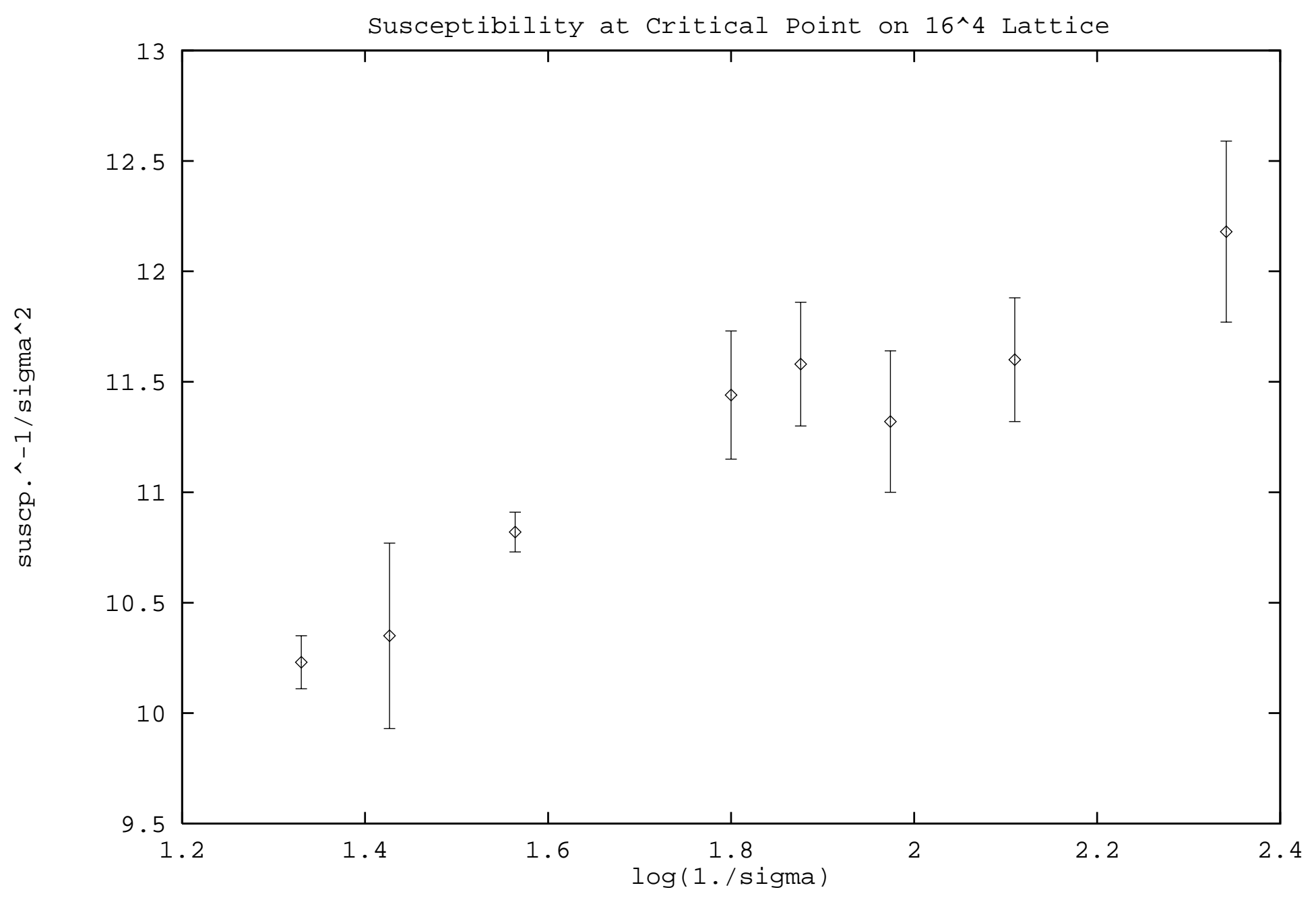




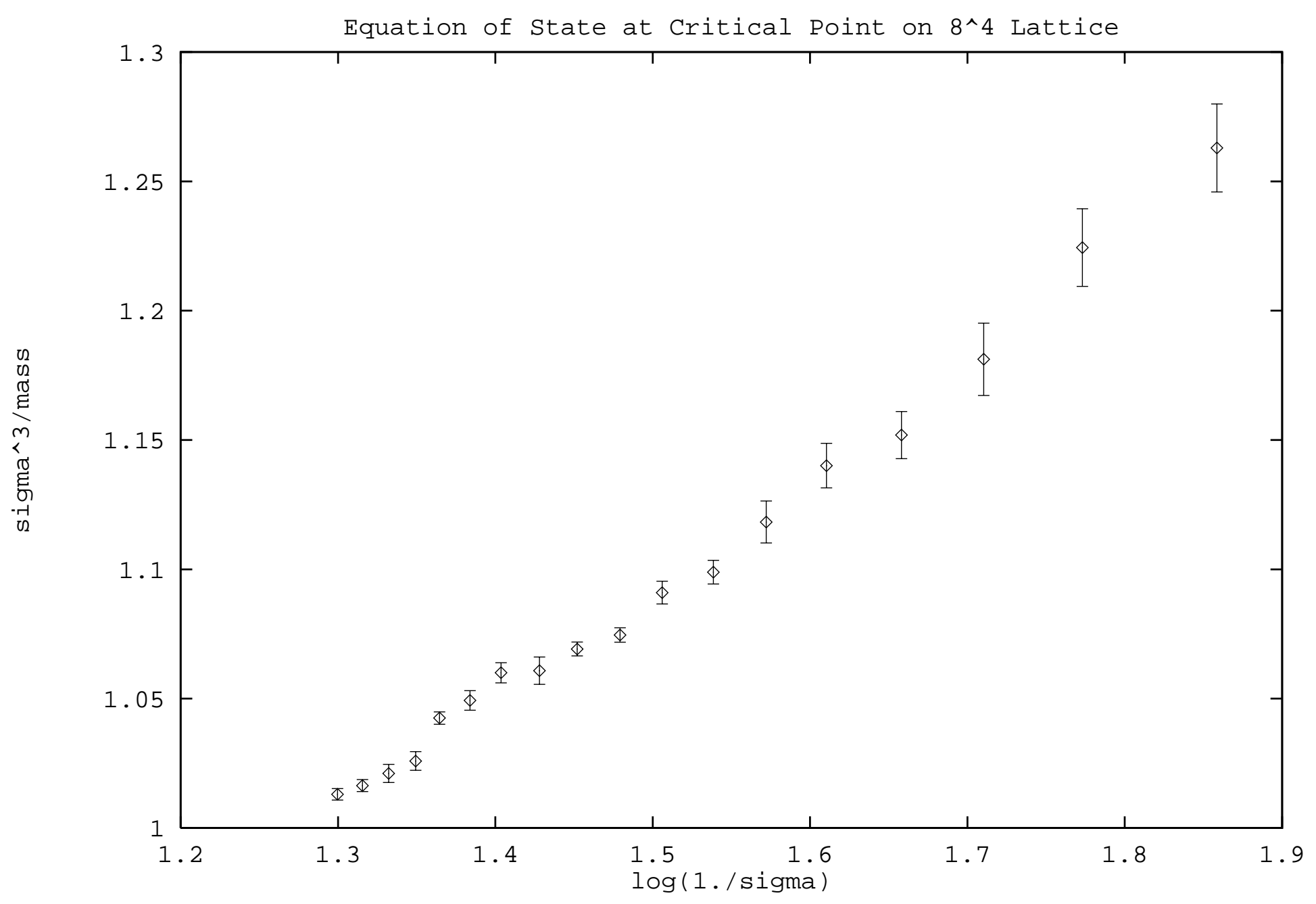




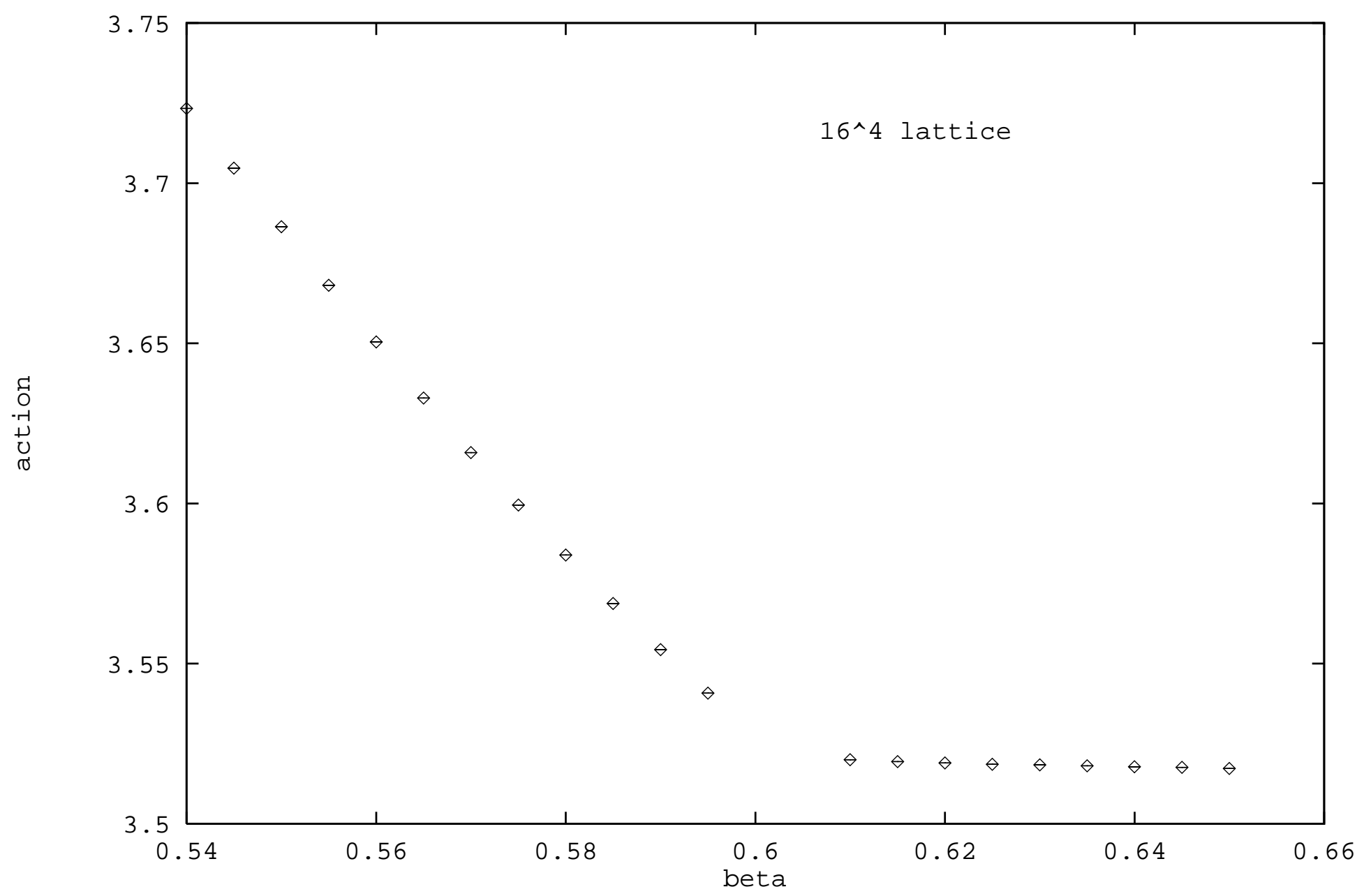




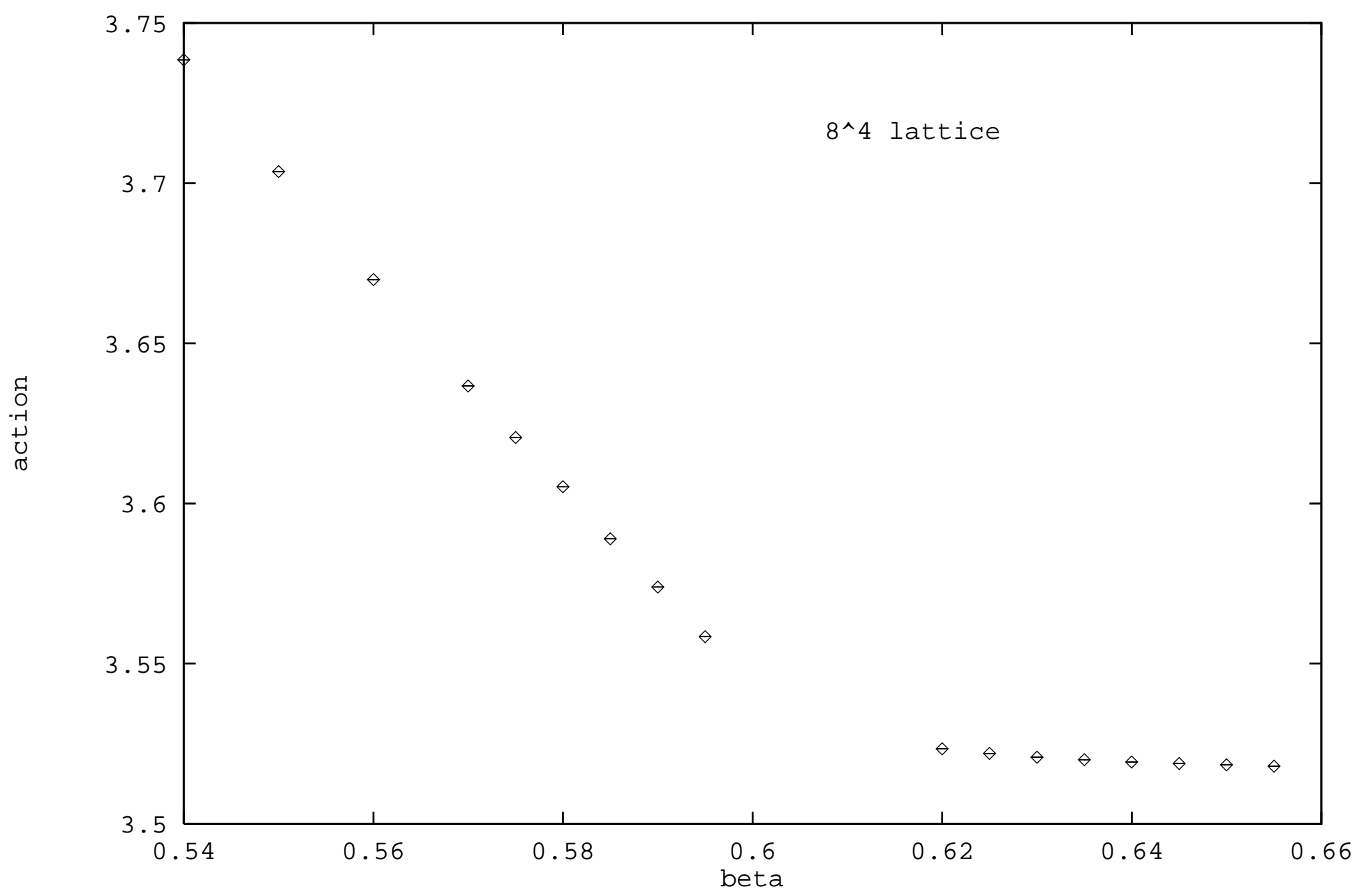




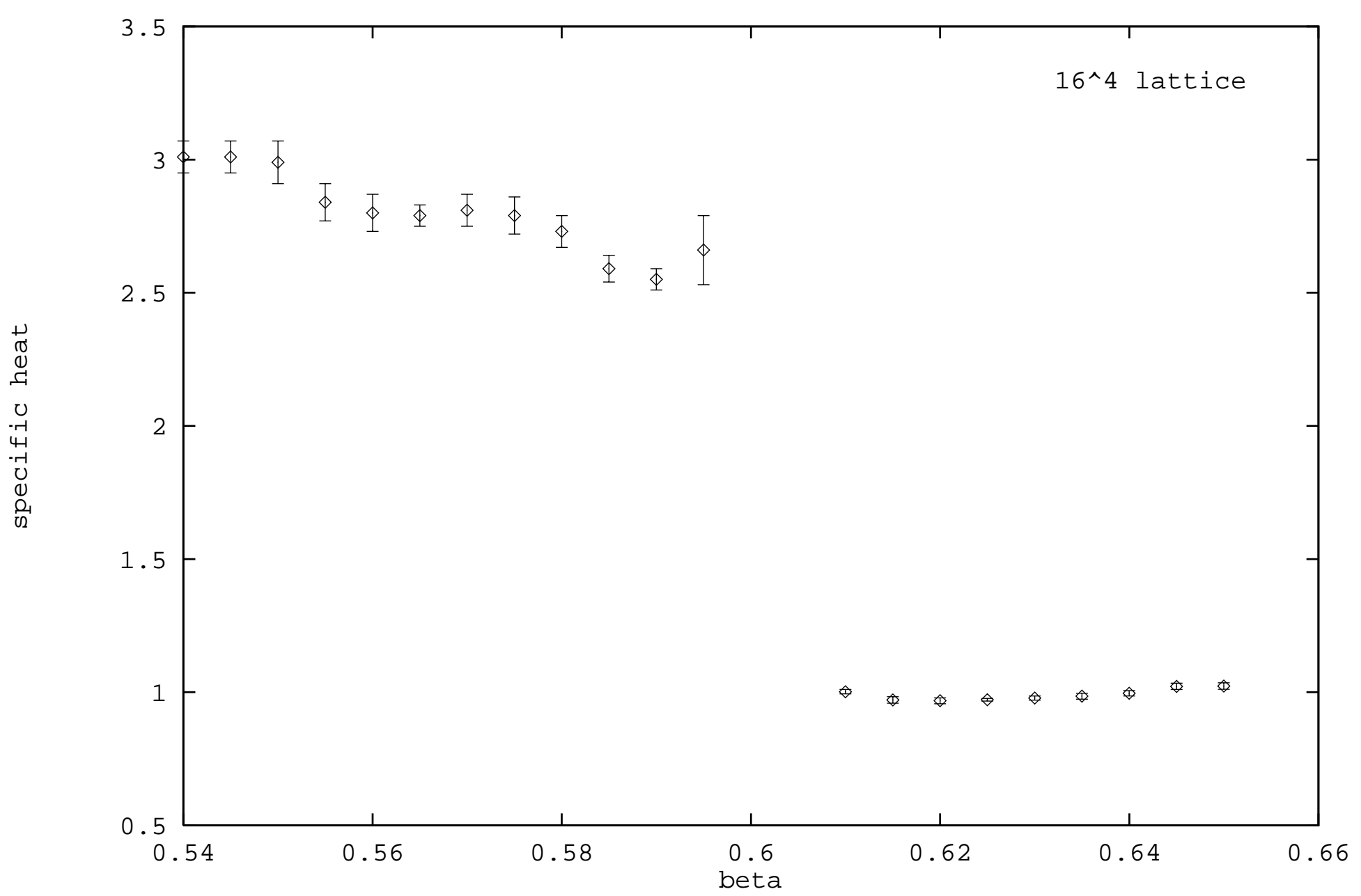




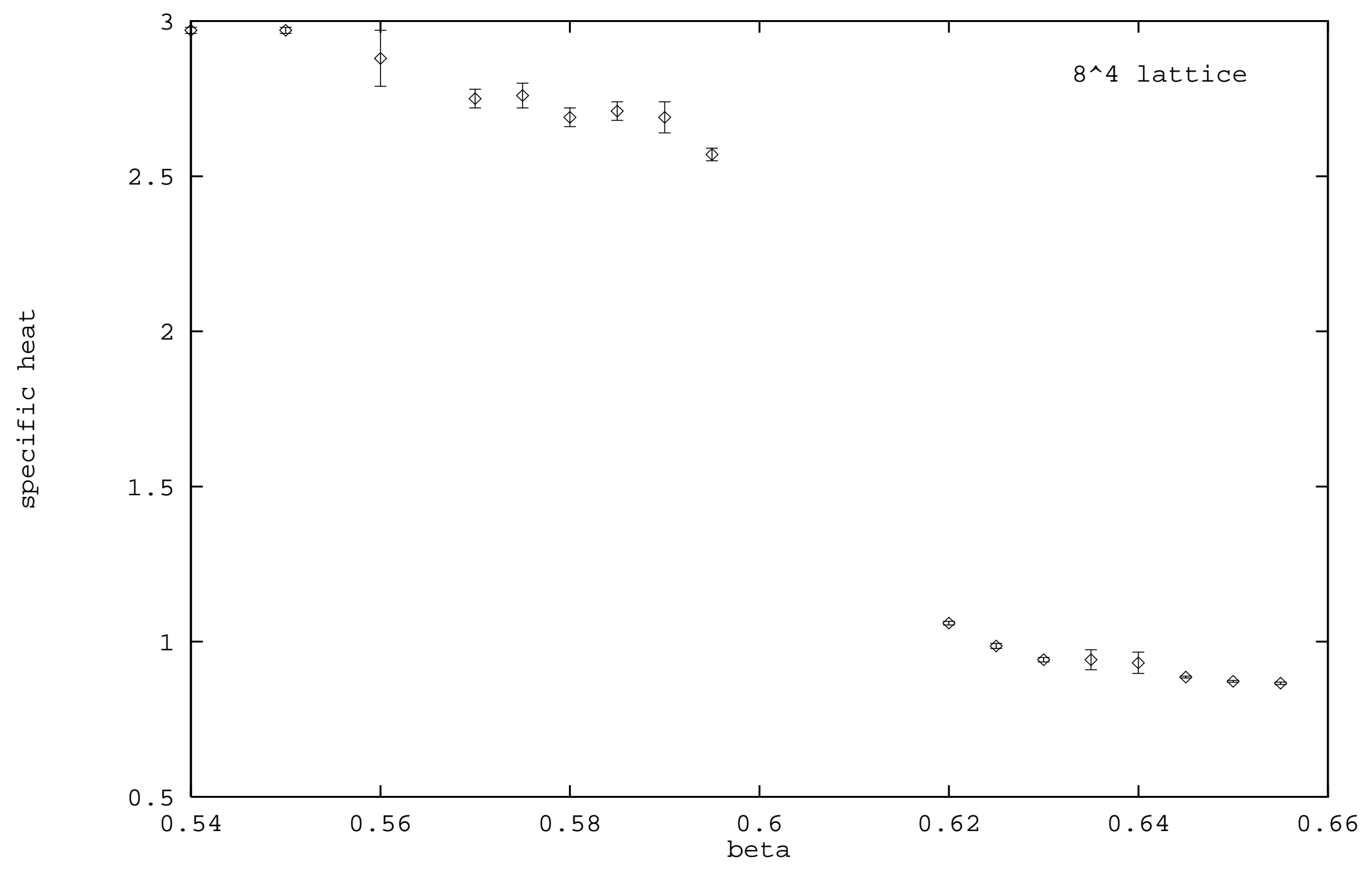

